\title{
ÉLOGE DE LA POLITIQUE
}

\author{
Charles KLEIBER
}

Elle n'a pas de nom. Elle a des difficultés à se tenir debout, un regard fixe, des tremblements et une grande sensibilité au bruit. Ainsi la décrit Colin Whitaker, vétérinaire à Ashford dans le Kent. C'est le 25 avril 1985. Elle n'a pas de nom ; on l'appellera plus tard la vache folle. C'est, Mesdames et Messieurs, à partir de son histoire ${ }^{1}$, c'est grâce à elle, pour nous et pour nos frères ruminants, que j'aimerais, me livrer à un bref éloge de la politique. Plus précisément : j'aimerais montrer que si l'homme, comme disait Ferdinand Gonseth, «ne peut être qu'en devenant ${ }^{2}$ », seule la pratique politique peut orienter nos innombrables devenirs possibles et leur donner un sens qui s'inscrive dans un projet civilisateur.

1986 : Plusieurs dizaines de cas de vaches folles sont signalés. Les premiers rapports qui les recensent admettent l'émergence d'une nouvelle maladie animale, et la classe dans la famille des encéphalopathies spongiformes - scrapie chez le mouton, CreutzfeldtJakob chez l'homme - qui aboutit invariablement à la dégénérescence du système nerveux. L'issue est fatale et atroce.

Tous ces rapports sont muets sur les causes et les modes de transmission de la maladie. Les conséquences possibles pour l'homme ne sont pas à l'ordre du jour et aucune mesure n'est évoquée, aucune instruction n'est donnée pour détruire les carcasses contaminées qui entrent, comme si de rien n'était, dans la chaîne alimentaire. Car la vie des vaches folles n'est pas terminée : elles deviendront de la farine industrielle fabriquée à partir de leurs détritus rachetés aux abattoirs. Ainsi inocule-t-on la maladie à des bovins sains. Ainsi les ruminants deviennent-ils carnivores.

Une voix, une seule, dénonce : celle du professeur Richard Lacey, spécialiste de microbiologie clinique à l'université de Leeds. Il parle déjà de « passivité criminelle ». Nous sommes en 1986, tout va bien.

1987 : 420 cas de maladie sont officiellement recensés. Les pistes convergent sur la nourriture des bovins. Le coupable est désigné : une dérégulation décidée par le gouvernement Thatcher qui permet aux équarrisseurs de réaliser des économies d'énergie et de réduire les coûts en limitant les températures de traitement des déchets animaux. L'agent infectieux est ainsi préservé. Pourtant, la raison d'État trouve cette année son

1. D'après CoJEAn (Annick) et FotTorino (Éric), « Chronique d'une négligence d'État », Le Monde, 6 avril 1996.

2. Gonseth (Ferdinand), Éditorial, Dialectica, 1, 1947, p. 7. 
discours qui ne variera plus : l'homme ne court aucun danger puisque la scrapie existe depuis deux cents ans sans dommage pour quiconque. Tout va bien.

1988 : 2185 bovins malades sont recensés. Le gouvernement britannique désigne en mai un groupe de travail présidé par sir Richard Southwood, professeur de zoologie à Oxford, très éloigné comme ses trois collègues de l'étude des maladies spongiformes. On le dit « compréhensif » à l'égard des impératifs d'État. Le groupe de travail propose néanmoins une première recommandation de bon sens que le gouvernement adopte : détruire les carcasses des animaux infectés. Mais il fixe la compensation à $50 \%$ seulement du prix des animaux détruits. Le gouvernement empêche ainsi, par souci d'économie, la destruction scrupuleuse des carcasses infectées. Les ruminants sont interdits de farine carnée, tandis que les cochons et les poulets conservent leur régime. Les consommateurs, eux, continuent de manger la viande infectée. Tout va bien.

1989 : 7136 cas de maladie bovine sont confirmés. Le comité Southwood publie son rapport et le gouvernement britannique respire. Le comité, en effet, affirme que la maladie n'est transmissible ni de vache à vache, ni de vache à veau. Puisque la farine carnée - vecteur officiel de la transmission - est désormais interdite, l'épidémie s'éteindra naturellement. Le rapport précise même la date (1996) et le nombre maximum d'animaux atteints (20 000). À quoi bon procéder aux abattages massifs réclamés par quelques esprits chagrins dont le professeur Lacey ? Les risques pour la population? Invérifiables avant dix ans, infimes pour ne pas dire nuls. Tout va bien.

Pourtant une sourde inquiétude demeure, alimentée par la croissance des chiffres. Un nouveau comité scientifique, présidé par le $\mathrm{D}^{\mathrm{r}}$ Alan Tyrell, est désigné, qui s'interroge enfin sur les possibilités de transmissions verticales et horizontales de la maladie et demande la mise en œuvre urgente d'une étude. Il s'agit d'examiner tous les cas signalés en Grande-Bretagne de la maladie de Creutzfeldt-Jakob ; il s'agit aussi de préciser la proportion des vaches atteintes par la maladie sans manifestation de symptômes et qui - de ce fait - entrent, malgré tout, dans la chaîne alimentaire. Mais le coût de cette étude est jugé excessif. D'ailleurs, précise le gouvernement, pourquoi étudier puisque l'extinction naturelle de la maladie est certaine. Tout va bien.

1990 : 14180 cas de maladie sont recensés. Après les vaches, les moutons, les bisons, un chat siamois, devenu fou, meurt à Bristol : la famille britannique est atteinte. Alors la rumeur gronde, les médias se font plus exigeants, l'inquiétude devient pesante. Le gouvernement, prisonnier de sa logique, n'a qu'une attitude possible : rassurer. Il rassure donc et la fille du ministre de l'Agriculture, Cordelia, quatre ans, mange innocemment un hamburger devant les caméras de la télévision.

Peu à peu, un cordon sanitaire se met en place. Le 30 mai, la France décrète un embargo sur la viande bovine, suivie par l'Allemagne et par le Luxembourg puis par la Belgique et le Portugal. Le débat passe du sanitaire à l'économique et au politique : la commission de l'Union européenne, qui endosse les thèses britanniques, menace de prendre des sanctions légales contre ces pays et de les poursuivre devant la Cour européenne de justice pour non-respect du principe de libre circulation des marchandises. Un compromis momentané est trouvé grâce aux engagements britanniques de renforcer le contrôle sanitaire du cheptel et de la viande. Le 6 novembre, la Suisse interdit la nutrition des ruminants avec la farine animale.

C'est à la fin de cette année qu'une expérience permet d'établir la possibilité de transmission de l'encéphalite spongiforme bovine au porc. Ce fait, essentiel, dément la 
thèse de la non-transmission de la maladie. Vu la troublante proximité organique entre l'homme et le porc, c'est, dit le professeur Lacey, « une très mauvaise nouvelle ». Rien ne va plus.

1991 : 25025 cas d'encéphalite spongiforme bovine sont reconnus. Surtout, un veau né après la date de retrait des aliments incriminés est reconnu porteur de la maladie. L'incertitude sur le mode de transmission augmente. L'Angleterre pourtant persiste : elle suit la science. Elle accepte le risque. Le doute ne profite pas au consommateur ; il bénéficiera pour l'instant aux producteurs et à ceux qui les défendent.

On connaît la suite : 35045 cas reconnus en Grande-Bretagne en 1992, 36755 en 1993 puis les chiffres diminuent. Mais les interdictions d'exportation se multiplient, le doute croît, la tension monte avec l'annonce que deux fermiers anglais ayant passé leur vie dans des exploitations laitières sont morts de la maladie de Creutzfeldt-Jakob. Le modèle historique de l'alimentation animale est mis en question. Si le mode de transmission de la maladie n'est toujours pas établi, l'hypothèse d'une transmission à l'homme se renforce. Aucune étude sérieuse n'est faite. Et pourtant, dit, avant d'autres, le Premier ministre anglais John Major, ce n'est pas la vache, c'est la presse qui est folle.

1996 : dix ans après la découverte de l'encéphalite spongiforme bovine, la preuve de sa transmission à l'homme, sous forme d'une variante mortelle de la maladie de Creutzfeldt-Jakob, est enfin établie. Mais l'interdiction complète des farines animales n'est toujours pas prononcée.

2000 : le gouvernement de Tony Blair publie le rapport de lord Nicholas Phillips of Worth Matravers. Trois ans d'enquête impitoyable pour mettre en lumière dix ans d'erreurs et de manipulations de l'opinion. Enfin, John Major présente ses excuses et le gouvernement travailliste décide d'indemniser les victimes. Mais, avec de nouveaux cas recensés, le débat s'enflamme en France et en Allemagne principalement. La Grande Peur du bœuf se propage partout en Europe, imposant des mesures symboliques d'apaisement pas toujours raisonnables et sollicitant plus que jamais la science.

Aujourd'hui le mal court toujours dans la campagne anglaise et sans doute dans les nôtres. De nombreux animaux continuent d'absorber des farines carnées. Le silence, les petites lâchetés ordinaires, les égoïsmes, les rivalités imbéciles ont tué ; elles tueront davantage. Selon les prévisions les plus actuelles, c'est quelques milliers de personnes ou quelques dizaines de milliers - 150000 ? - qui pourraient mourir après une période d'incubation de plusieurs dizaines d'années. Peut-être «beaucoup, beaucoup plus », ajoute un ministre britannique. Telle est jusqu'à ce jour l'histoire de la vache folle, ses incertitudes et ses questions.

Derrière le bruit, derrière les silences, derrière les drames de cette sale histoire, derrière ces morts annoncées, une question ne cesse de nous interpeller : qu'est-ce qui a guidé les comportements de ceux qui, volontairement ou involontairement, consciemment ou inconsciemment, l'ont écrite ?

La science ne guide plus. Le professeur Southwood, le docteur Tyrell, le professeur Lacey même, et les autres, tous ont donné leurs versions de la vérité, posé leurs questions, donné leurs réponses, formulé leurs doutes. Que faire de tant d'incertitudes, d'hypothèses, de vérités contradictoires orientées à l'évidence par le vécu et les enjeux personnels. Que faire des connaissances nouvelles quand ces connaissances deviennent des facteurs 
de production nécessaire à la compétitivité des entreprises et à la prospérité des nations ? Comment trouver le juste chemin quand les enjeux scientifiques, économiques et politiques sont inextricablement liés ? Comment transformer la connaissance en sagesse ? Le gouvernement britannique a choisi ses experts, puis s'est porté garant de leur science, celle qu'il a bien voulu financer. La preuve par les faits et la raison a montré ses limites. Peut-on mieux démontrer que la science n'est plus cette raison commune, ce langage universel et incontesté qui apporte des réponses aux questions des hommes?

Les religions : elles ne guident pas davantage. Depuis longtemps à travers les morts de l'intolérance et tous les fondamentalismes, elles ont montré leur impuissance à faire vivre les hommes entre eux. On le savait : la preuve par l'amour des religions ne prouve plus grand-chose dans un monde de plus en plus individualisé, où chacun, seul ou presque, construit ou plutôt bricole son système de significations, choisit ses mythes et ses croyances.

Qu'est-ce qui nous guide ? Le marché, disent-ils, institution imparfaite et nécessaire, la plus mauvaise à l'exception de toutes les autres... Certainement pas celui, qui sous prétexte de compétitivité économique, a été déréglementé au point d'ignorer l'exigence de stérilisation des déchets carnés, d'oublier les contrôles d'hygiène des abattoirs et finalement de tuer. Certainement pas celui qui n'a d'autre ambition que la rationalité des échanges et la rentabilité à court terme, certainement pas celui, orgueilleux et dominateur qui se déploie au niveau de la planète et fait dire à certains que les marchés gouvernent et que les gouvernements gèrent. Ce marché-là, porteur d'aucune ambition sociale et d'aucun rêve, ne peut guider une communauté. Il ne peut qu'orienter les comportements économiques et répartir les ressources et le profit. Mais peut-être certains marchés pourraient-ils être conçus pour tenter de réconcilier efficacité économique et responsabilité écologique, le court terme et le long terme. Ceux-là prendraient en compte les coûts de la pollution, du chômage, des atteintes à l'environnement et à la santé ; les prix du marché comprendraient un montant compensatoire suffisamment faible pour ne pas freiner la compétition économique et suffisamment fort pour responsabiliser directement l'entreprise sur les conséquences sociales et écologiques de sa performance. Ce marché-là, qui serait un outil économique et simultanément politique d'allocation de ressources pourrait peut-être être guidé. Il reste à construire et c'est une autre histoire.

L'éthique peut-elle guider ? L'histoire de la vache folle permet d'en douter. Tous, au cours de cette tumultueuse affaire, les scientifiques, les éleveurs, les gouvernements, les consommateurs, tous auront évoqué pêle-mêle la liberté d'entreprendre, l'efficacité économique, le principe de précaution, la liberté académique, le bien des malades, la protection des consommateurs, le secret médical ; tous auront mis le citoyen, le consommateur, le producteur, la science, les malades, les animaux au centre de leur préoccupation, avec les résultats que l'on sait. Conclusion : l'éthique indolore et acratopège, l'éthique abstraite et rassurante de l'Homme, du droit, l'éthique minimale de l'évitement du mal et de la douleur - ce qu'Henri Atlan appelle l'éthique de l'enfant -, cette éthique ne guide pas. Elle fixe des limites, parfois empêche l'impensable, mais justifie aussi l'injustifiable en offrant à chacun - pas seulement aux instances politiques - un alibi. L'éthique institutionnelle ne guide pas ; elle protège les institutions. Elle ne peut penser le bien de tous. Au mieux, peut-elle désigner le mal : ce sera ce qui menace les institutions. 
Plus précisément : elle ne peut travailler à l'avènement d'un avenir nouveau et encore insoupçonné puisqu'elle admet au départ que l'homme est une victime passive, d'un malheur dont il faut le protéger. Elle ne peut inventer; elle préserve.

Pourtant il y a eu le professeur Lacey. Lui, pendant dix ans, n'a cessé d'exiger la vérité. À partir de sa conception des faits, à partir de ses recherches et de son expérience, cet homme tranquille et plutôt conservateur, a harcelé de ses questions le gouvernement et les commissions scientifiques successives. Apparaît à travers lui, sans doute à travers d'autres, une conception différente de l'éthique. Il s'agit non pas de l'éthique abstraite et passive des grands principes et des institutions mais celle, individuelle et plus fragile, des situations concrètes. Il s'agit d'une recherche, parfois d'une ascèse, d'un long effort de mise en ordre relationnelle, d'une tentative de dégager des exigences pratiques par rapport à une idée momentanée de la vérité, ou plutôt : d'un consensus possible, sur une vérité provisoire. Cette éthique-là ne préserve pas, elle invente.

L'exigence de vérité, la passion de comprendre : elles ont amené le professeur Lacey à ne pas accepter comme inéluctable la raison commune qui aurait pu justifier de ne pas aller jusqu'au bout, d'abandonner, de ne pas comprendre, de ne pas dénoncer avec tout ce qui est disponible et sans rien considérer d'autre. Il aura fallu pour cela un engagement complet dans le réel de la situation. Il aura fallu maintenir une insatisfaction radicale et protéger la capacité de rébellion comme cette petite flamme vacillante qui éclaire la nuit et qui constitue « la part immortelle de l'homme ${ }^{3}$ ». C'est dans cette longue quête qui le met tout entier en question, que l'homme devient.

Mais les professeurs Lacey ne sont pas si nombreux. Nous voilà donc sans connaissance unifiée, sans raison commune, sans religion, presque sans éthique, juste avec une petite flamme pour nous dire, par-delà l'air du temps, au-delà des apparences et des clichés, comment est le monde et comment il faut se comporter pour être en harmonie avec lui. L'histoire de la vache folle le montre : le progrès dont l'idée nous habite depuis deux siècles n'aboutit pas nécessairement aux retrouvailles de l'homme et de la nature. La vérité se dérobe : elle est plurielle, complexe, changeante. Elle déserte parfois ce monde, devenu village et ces institutions dans lesquelles tant bien que mal nous tentons de vivre et de travailler.

Qu'est-ce qui nous guide ? On pourrait dire, plus généralement : qu'est-ce qui guide le changement de nos sociétés et oriente nos rêves ? Qu'est-ce qui guide la destruction créative des paradigmes, des idées, des emplois, de nos économies, des représentations de nous-mêmes et du monde, et tout ce qui nous emporte nul ne sait où ?...

Pour terminer, j'aimerais, dans un bref hommage de la politique, montrer que, malgré sa crise de légitimité, malgré son effacement progressif devant l'économique, guider est la fonction principale de la politique et qu'elle seule pourrait, à certaines conditions, le faire.

Guider : ce terme est contestable. Il s'agit plutôt de gouverner, c'est-à-dire de rendre auteur. On pourrait dire aussi : faire du gouverné quelqu'un capable de faire œuvre de lui-même, d'inventer sa vie et peut-être de penser sa fin. Vaste programme, qui pose toute la question du renouvellement du modèle de la gouvernance publique, de ses concepts et de ses instruments hérités au mieux du XIX siècle. Quelle conception de la

3. BADIOU (Alain), L'Éthique, essai sur la conscience du mal, Paris, Hatier, 1993. 
gouvernance peut être à la hauteur de l'enjeu ? Quand tous les jours nous inventons de nouvelles technologies qui en retour nous inventent, quand les interdépendances économiques, écologiques et culturelles transforment les questions intérieures en questions planétaires, quand l'économie se désinsère du social et du culturel et s'autonomise toujours davantage, quand la logique du toujours plus et les mythes de la démesure - mythe de la santé parfaite et de l'éternelle jeunesse, mythe de Prométhée maîtrisant la nature se renforcent et créent sans cesse de nouveaux « besoins », quand les finalités deviennent floues, il y a danger, il y a urgence : l'histoire de la vache folle en témoigne. La politique ne peut plus être la simple mise en forme de l'inéluctable, une signature sur l'air du temps qui accompagne le grand fleuve tranquille du changement. Il faut inventer de nouvelles formes de gouvernance, il faut repolitiser la politique. Comment?

D'abord, première exigence, en garantissant, ici et maintenant et quel qu'en soit le prix, le fondement du « vivre ensemble » : la sécurité, la santé publique, l'état de droit et ses libertés, la transparence, le débat. Tout est à craindre sans ce socle de droits démocratiques et sa permanente adaptation. Combien, dans l'histoire de la vache folle, paieront ses fissures de leur vie?

Ensuite, deuxième exigence, en participant activement à la construction d'un ordre public international, seule réponse à la mondialisation, seul moyen capable d'assurer un développement durable et de garantir le partage de la richesse et du savoir, donc la paix, sur notre belle et unique planète. Ce cadre législatif transnational sera l'instrument principal de la gestion de l'espace à grande échelle et le fondement de l'action à long terme. Car la maîtrise des grands espaces et de la longue durée est désormais une obligation qui dépasse les individus vivants, identifiables et prévisibles. Les choix collectifs, l'histoire de la vache folle à nouveau en témoigne, concernent de plus en plus les générations futures et la communauté des nations, à qui les patrimoines construits dans la durée sont transmis. Tout cela suppose la construction progressive et patiente d'une conception transnationale et transgénérationnelle du bien commun. Vaste programme...

Troisième exigence, en protégeant le service public. Non pas l'administration de prestations par des appareils administratifs insensibles, à des administrés passifs, justiciables, patients, étudiants, laissés-pour-compte, vieux, pauvres, étrangers abandonnés, filles mères, femmes battues..., mais le service au public - c'est-à-dire la construction d'une économie publique avec ses savoirs, ses exigences de qualité, ses mécanismes de dépassement et de concurrence organisée, ses entrepreneurs, ses entreprises, sa culture, sa fierté, sa capacité de s'inventer et de rendre des comptes.

Enfin, quatrième exigence, en acceptant l'impuissance d'ordonner la société et le changement et en créant les conditions qui permettent au plus grand nombre d'inventer l'avenir et de mettre de la vie dans les processus démocratiques, de la vie et rien d'autre. De la vie ou ce qui naît du choc des valeurs différentes, de la confrontation de vérités contradictoires puis de la tentative de les réconcilier. C'est peut-être le plus urgent et le plus difficile. À travers la multitude des arbitrages, à travers les innombrables compromis qui nous permettent de vivre ensemble, se construiraient peu à peu un projet explicite, une aventure toujours en devenir, portée par une démarche mutuellement pédagogique. La politique serait alors cela : une manière nouvelle de dire nos contradictions et de les transformer en action, la capacité d'individualiser l'action collective et de donner la parole à tous les professeurs Lacey et à ceux - rebelles, emmerdeurs, empêcheurs - qui représentent la conscience critique de la société. Ce serait la volonté 
tenace d'écouter, de comprendre pour agir et d'agir pour comprendre, le courage de choisir, donc de renoncer, pour ajuster une attente sociale infinie à des ressources limitées. Ce serait le débat permanent sur les conditions concrètes d'un «vivre ensemble » et surtout, la construction patiente, par la formation et la vie en société, de l'autonomie et de la responsabilité individuelles. Il faut pour cela que la politique soit présente là où la société s'élabore, qu'elle repose sur une idée et un goût du bonheur, qu'elle s'enracine dans la culture et que les femmes et les hommes qui s'y engagent reconnaissent dans l'autre les multiples visages de la vérité. Il faut une ambition qui se nourrit dans l'action, par l'échec et par le succès, et se construit patiemment, les pieds dans la boue et la tête dans les étoiles.

Si notre espace est de plus en plus indifférencié, sans frontières, sans normes, sans séparation entre l'interdit et le permis, il faut que la politique, guidée par une conception du juste et de l'injuste, du vrai et du faux, recrée du sens et montre dans les faits que l'innovation scientifique, technologique, économique, culturelle, sociale et politique vont ensemble. Cette politique reste à inventer; elle sera en devenant.

Ces quelques exigences sont à mon sens nécessaires pour nous « rendre auteurs ». Sans doute ne sont-elles pas suffisantes. Vous pourriez, Mesdames et Messieurs, en suggérer bien d'autres. J'en ajoute une, exigée par l'esprit des temps et cet acharnement collectif au pragmatisme qui n'a d'autre objectif que l'arrangement à court terme. Une exigence qui pourrait nous aider à penser le changement plutôt en terme de chance que de menace ; une exigence qui s'appuierait sur un empirisme savant et sur la connaissance approfondie du terrain. Une exigence ? - Juste un vœu : l'utopie, celle qui s'appuie sur la réalité, celle qui crée l'élan et le transforme en désir d'avenir.

Charles KLEIBER* (novembre 2000).

\footnotetext{
* Charles KLEIBER, architecte, directeur des hôpitaux universitaires du canton de Vaud, enseignant en économie de la santé à l'université de Lausanne, est secrétaire d'État à l'éducation et à la recherche (Secrétariat d'État à l'éducation et à la recherche, 4 Hallwylstrasse, CH-3003 Berne, Charles.kleiber @ sbf.admin.ch).
} 